\section{Impact of amblyopia on vision at age 12 years: findings from a population-based study}

D Robaei ${ }^{1}$, A Kifley ${ }^{1}$, KA Rose ${ }^{2}$ and P Mitchell ${ }^{1}$

Introduction

Aims To report prevalence of amblyopia and long-term impact of its treatment on vision in a population-based sample of 12-year-old Australian children.

Methods Logarithm of minimum angle of resolution (logMAR) visual acuity (VA) was measured in 2353 children (response rate $75.3 \%$ ); visual impairment was defined as $\mathrm{VA}<6 / 12$. Amblyopia was defined using various criteria of best-corrected VA, together with an amblyogenic factor and absence of significant organic pathology. Corroborative historical data on previous diagnosis and treatment were obtained from parental questionnaires.

Results Forty-four children (1.9\%) were diagnosed with amblyopia, unilateral in 40 and bilateral in four. Isolated anisometropia was the most frequent cause $(\mathbf{4 1 \%})$, followed by strabismus (25\%), combined anisometropia and strabismus (23\%), and high ametropia (9\%). Myopia, hyperopia, and astigmatism were present in 28,51 , and $44 \%$ of amblyopic children, respectively, compared to 12,4 , and 9\% of non-amblyopic children. Mean bestcorrected VA in amblyopic eyes was 44.5 logMAR letters (Snellen equivalent 6/9), range: 11-60 letters. Most children with amblyopia (84\%) had been treated. Only $27 \%$ were visually impaired in their amblyopic eye. Conclusions This report documents a low amblyopia prevalence in a population of 12year-old Australian children. Amblyopic visual impairment was infrequent in this sample despite absence of mandatory vision screening. Eye (2008) 22, 496-502; doi:10.1038/sj.eye.6702668; published online 23 March 2007

Keywords: children; epidemiology; amblyopia; Sydney Myopia Study; Sydney Childhood Eye Study
Amblyopia is an important cause of noncorrectable unilateral visual impairment in industrialised countries ${ }^{1}$ despite knowledge that amblyogenic risk factors are easily detectable by trained personnel, and that treatment is well tolerated ${ }^{2,3}$ and highly effective $^{4}$ when instituted within the sensitive period of development.

Snowdon and Stewart-Brown's 1997 review of the effectiveness of preschool vision screening identified paucity of data on the natural history of amblyopia and the extent of disability attributable to it. ${ }^{5}$ Although there are substantial data on the prevalence of amblyopia from different populations, few studies have examined long-term impacts of amblyopia detection and treatment on vision in population-based samples. ${ }^{6,7}$

The Australian population benefits from a universal healthcare system covering most costs of both optometric and ophthalmic consultations; preschool vision screening, however, is now not routinely performed. The detection of amblyopia and associated risk factors therefore depends on parental selfreferral and a high degree of suspicion by primary healthcare providers.

Given this context, we recently reported findings on visual impairment and amblyopia in a large population-based sample of 6-yearold Australian children. ${ }^{8,9}$ The overall prevalence of amblyopia was relatively low in that younger population (1.8\%), and most children with amblyopia (72\%) had already been diagnosed and treated; newly diagnosed children were directly referred to paediatric ophthalmologists for further management. The Paediatric Eye Disease Investigator Group (PEDIG) recently demonstrated significant response to treatment (defined as visual acuity 
improvement of at least two lines) in over half of amblyopic children aged 7-12 years. ${ }^{10}$ Therefore, 6-year-old children, such as those newly diagnosed with amblyopia in our previous sample stand to gain significant benefit from treatment if promptly instituted.

This second report documents the impact of amblyopia on vision in a population-based sample of predominantly 12-year-old Australian children, representing an age beyond which treatment of amblyopia is unlikely to result in any substantial visual acuity improvement. ${ }^{11}$ It therefore documents the likely extent of permanent visual disability associated with amblyopia, and permits appraisal of outcomes in the absence of routine preschool vision-screening programmes.

\section{Materials and methods}

\section{Population}

This project is a population-based survey of refraction and other eye conditions in two samples of school children predominantly 6 and 12 years of age, resident in the Sydney metropolitan area. ${ }^{8,9,12-14}$ It forms part of the Sydney Childhood Eye Study, which is examining childhood eye conditions across a range of ages. Methods used to identify and select the target sample, as well as a description of study procedures have been reported. ${ }^{12}$ In brief, the study area was stratified by socio-economic status to randomly select 34 primary and 21 secondary schools from a proportional mix of public and private/ religious schools across Sydney. The following report is based on data from the 12-year-old sample of children examined from 2004 to 2005.

\section{Procedures}

Written informed consent from at least one parent plus assent of each child was obtained before examination. Approval for the study was obtained from the Human Research Ethics Committee, University of Sydney and Department of Education and Training, state of New South Wales, Australia.

Distance visual acuity was tested monocularly using a logarithm of the minimum angle of resolution (logMAR) chart. The chart was retro-illuminated with automatic calibration to $85 \mathrm{~cd} / \mathrm{m}^{2}$ (Vectorvision CSV- $1000^{\mathrm{TM}}$, Dayton, OH, USA) and read at eight feet $(244 \mathrm{~cm})$. Visual acuity (VA) was assessed with and without spectacle correction, if worn. ${ }^{8}$ Children with presenting VA $<0.02$ $\log$ MAR units (i.e. $<54$ letters, equivalent to Snellen acuity <6/6) underwent subjective refraction according to the Beaver Dam Eye Study modification of the Early Treatment Diabetic Retinopathy Study protocol. ${ }^{15}$ Using a VA cutoff of 6/12, uncorrected and presenting visual impairment were defined by unaided and spectacle-corrected VAs, respectively. Non correctable visual impairment referred to $\mathrm{VA}<6 / 12$ after subjective refraction.

Cycloplegia was obtained after one cycle of cyclopentolate $1 \%$ (one drop) and tropicamide 1\% (one drop), following corneal anaesthesia with amethocaine hydrochloride $1 \%$. In a small proportion of children slow to dilate, phenylephrine hydrochloride $2.5 \%$ was used to maximise mydriasis. Cycloplegia was considered full when the pupil was fixed and $\geqslant 6 \mathrm{~mm}$ diameter.

An autorefractor (Model RK-F1, Canon, Japan) was used to perform cycloplegic autorefraction and keratometry. Children had a comprehensive eye examination comprising prism bar cover testing at near $(30 \mathrm{~cm})$ and distance $(6 \mathrm{~m})$ fixation, assessment of ocular movements and stereopsis using the TNO test, ocular biometry, slit-lamp examination, optical coherence tomography, and mydriatic digital retinal photography.

Parents completed a comprehensive 173-item questionnaire, and children completed a smaller survey. Socio-demographic information covering ethnicity, country of birth, parental education, and occupations was included. Parental education was defined as the highest level of education completed by either parent. This ranged from never having attended school to having completed a higher degree, such as a Masters or PhD. Socioeconomic status was based on home ownership by the child's parents and their employment status.

\section{Ascertainment of amblyopia cases}

In order to capture all children with amblyopia, including those with previously detected (and treated) amblyopia, a rigorous set of inclusion criteria were applied stepwise:

1. Criterion $A$-All children with best-corrected $\mathrm{VA}<6 / 6$ not attributable to organic disease of the eye or visual pathway were included as potential cases.

2. Criterion $B-$ All children with parent-reported diagnosis of amblyopia, occlusion, or atropine penalisation treatment (regardless of best-corrected VA) were also considered potential cases. These children were also required to be free from any organic disease of the eye or visual pathway.

3. Criterion $\mathrm{C}$-To be considered a definite case of amblyopia, children in either of the above two groups were required to have an amblyogenic factor demonstrable at the time of examination.

Anisometropic amblyopia was assigned as the cause if there was at least a 1.0 dioptre (D) difference in spherical equivalent refraction (SER) between the two eyes, in the absence of strabismus. Strabismic 
amblyopia was assigned as the cause if any heterotropia was present on cover testing or if there was confirmed history of strabismus surgery without anisometropia or high refractive error. Mixed amblyopia was assigned as the cause if the above causes were present in combination. Isoametropic amblyopia was assigned as the cause in the presence of bilateral hyperopia $>4.0 \mathrm{D}$, bilateral myopia $\leqslant-6.0 \mathrm{D}$ or bilateral astigmatism $\geqslant 2.5 \mathrm{D}$. Stimulus deprivation amblyopia was assigned as the cause if congenital cataract, ptosis or corneal or other media opacities obstructed vision, or if there was a confirmed history of these conditions.

4. Criterion D - If criteria A and C were met without criterion $B$ (previous diagnosis or treatment), children were only considered to have amblyopia if bestcorrected VA was less than 6/9. Thus, in cases with best-corrected VA $\geqslant 6 / 9$, parental report of previous treatment was required.

In uncertain cases, diagnosis and treatment were confirmed with the child's treating ophthalmologist.

\section{Data handling and statistical analysis}

Data were entered into a Microsoft Access database. Statistical analyses were performed using Statistical Analysis System (SAS Institute, Cary, NC, USA). Mixed models and generalised estimating equations adjusted for clustering within schools. Where cluster effects were insignificant, $\chi^{2}$ and $t$-tests were used. Sample means and mean differences are reported with their standard errors; all confidence intervals (CI) presented are 95\% CI.

\section{Results}

\section{Subjects}

Of 3144 eligible children, 2367 children were given parental permission to participate and questionnaires were provided by parents (response rate $75.3 \%$ ). Of these 2367 children, 14 were not examined because of absence from school during the examination period. The mean age of participants was 12.7 (range: 11.1-14.4 years); $49.4 \%$ were girls. Basic socio-demographic data on study participants are presented in Table 1. There was no significant gender or ethnic differences between responders and non-responders.

\section{Prevalence of amblyopia}

Using the above ascertainment criteria, 44 children (1.9\% of the sample) were diagnosed with amblyopia; 22 were boys. Using conventional criteria of best-corrected $\mathrm{VA}<6 / 12$ and a two-line difference between the eyes, the
Table 1 Selected socio-demographic characteristics of entire sample

\begin{tabular}{lccc}
\hline Characteristic & $\begin{array}{c}\text { All } \\
(\mathrm{n}=2353) \\
\mathrm{n}(\%)\end{array}$ & $\begin{array}{c}\text { Boys } \\
(\mathrm{n}=1190) \\
\mathrm{n}(\%)\end{array}$ & $\begin{array}{c}\text { Girls } \\
(\mathrm{n}=1163) \\
\mathrm{n}(\%)\end{array}$ \\
\hline $\begin{array}{l}\text { Age (years) } \\
11-11.99\end{array}$ & $100(4.3)$ & $46(3.9)$ & $54(4.6)$ \\
$12-12.99$ & $1645(69.9)$ & $787(66.1)$ & $858(73.8)$ \\
$13-13.99$ & $603(25.6)$ & $354(29.8)$ & $249(21.4)$ \\
$\geq 14$ & $5(0.2)$ & $3(0.3)$ & $2(0.2)$ \\
& & & \\
Ethnicity & & & \\
European Caucasian & $1407(60.0)$ & $740(62.5)$ & $667(57.5)$ \\
East Asian & $352(15.0)$ & $154(13.0)$ & $198(17.1)$ \\
Middle Eastern & $166(7.1)$ & $93(7.9)$ & $73(6.3)$ \\
South Asian & $129(5.5)$ & $60(5.1)$ & $69(6.0)$ \\
Mixed & $179(7.6)$ & $89(7.5)$ & $90(7.8)$ \\
Other & $112(4.8)$ & $49(4.1)$ & $63(5.4)$ \\
& & & \\
Parental education & & & \\
Secondary school & $684(33.3)$ & $320(30.7)$ & $364(35.9)$ \\
Technical college & $536(26.1)$ & $281(27.0)$ & $255(25.2)$ \\
University & $834(40.6)$ & $440(42.3)$ & $394(38.9)$ \\
Parental employment & & & \\
Both employed & $1183(57.0)$ & $622(59.1)$ & $561(54.8)$ \\
One employed & $695(33.5)$ & $337(32.0)$ & $358(35.0)$ \\
Other & $198(9.5)$ & $93(8.8)$ & $105(10.3)$ \\
& & & \\
Parental home ownership & & & \\
Yes & $1555(74.2)$ & $810(76.6)$ & $745(71.8)$ \\
No & $540(25.8)$ & $248(23.4)$ & $292(28.2)$ \\
\hline
\end{tabular}

${ }^{\mathrm{a} C o m p r i s i n g ~ P a k i s t a n i, ~ I n d i a n, ~ a n d ~ S r i ~ L a n k a n ~ e t h n i c i t i e s . ~}$

prevalence was $0.4 \%$. There were no significant ethnic differences in amblyopia prevalence, being 2.0\% (CI 1.1-2.9\%) in European Caucasian children, 2.6\% (CI 1.1-4.0\%) in East Asian children, 1.6\% (CI 0.0-3.3\%) in South Asian children, and $1.2 \%$ (CI 0.0-2.6\%) in Middle Eastern children. The right eye was affected in 17 cases (39\%), the left eye in 23 cases (52\%); four children had bilateral amblyopia.

\section{Causes of amblyopia}

Anisometropia was the most frequent cause, present in 18 cases $(41 \%)$, followed by strabismus in 11 cases (25\%), combined anisometropia and strabismus in 10 cases (23\%) and high ametropia in four cases (9\%). The last four children had bilateral amblyopia, owing to bilateral high myopia and high hyperopia, each for two cases. One child was considered to have amblyopia despite failing to meet criterion $C$ (presence of an amblyogenic factor). She had best-corrected VA of 0.6 logMAR (Snellen equivalent 6/24) and reduced stereoacuity $(240 \operatorname{arcsec})$, in the absence of organic disease of the eye or visual pathway. 


\section{Previous diagnosis and treatment}

Using parental questionnaire data, 39/44 children (89\%) had previously been diagnosed with amblyopia by an eye practitioner, and all but one reported previous treatment. The mean age at diagnosis was 4.3 years (CI 3.4-5.3), although the relevant information could not be confirmed in 10 children. Treatment consisted of spectacle prescription in 34 cases, occlusion therapy in 22 , and atropine penalisation in two cases. A single form of treatment (either spectacles or occlusion alone) was reported in half the cases, whereas a combination of spectacles, occlusion, and/or atropine penalisation was reported in the remaining half.

\section{Visual acuity outcomes in children with amblyopia}

Figure 1 outlines the distribution of best-corrected VAs for all children with previously treated and untreated amblyopia. Most children diagnosed with amblyopia (73\%) had no visual impairment $(\mathrm{VA}<6 / 12)$ in their affected eye. Of these 32 children without visual impairment, 28 had previously been treated. Conversely, of the six previously untreated children, only two were

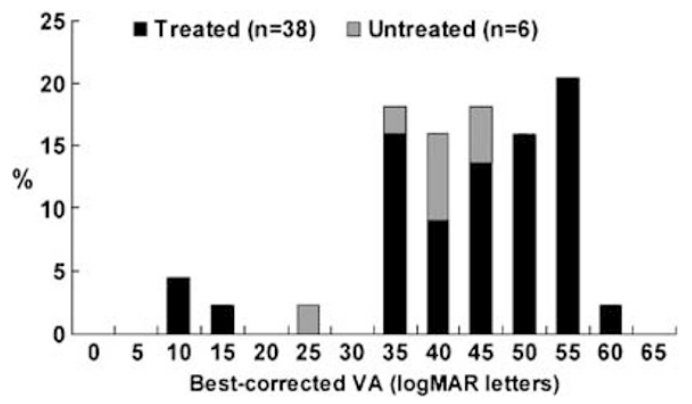

Figure 1 Distribution of best-corrected VA in 44 children with amblyopia. VA of the right eye is used in four cases of bilateral amblyopia. visually impaired in their amblyopic eye, with bestcorrected acuities of $6 / 24$ and $6 / 15$, respectively. Table 2 presents mean best-corrected acuities by the cause of amblyopia and outlines the proportion of visually impaired children in each category. All children with strabismic amblyopia had been treated, and they had the highest level of acuity, with a mean of $50.8 \log$ MAR letters (6/7.5 Snellen equivalent). Correspondingly, only one child with strabismic amblyopia had non-correctable visual impairment owing to amblyopia.

\section{Amblyopia and associated ocular outcomes}

Table 3 presents the prevalence of selected ocular outcomes for children with and without amblyopia. Figure 2 shows the distribution of spherical equivalent refraction in amblyopic eyes compared to right eyes of nonamblyopic children. Children with amblyopia had slightly more hyperopic spherical equivalent refraction, with a mean of $+1.47 \mathrm{D}$ compared to $+0.48 \mathrm{D}$ for children without amblyopia $(P=0.1)$, although myopia, hyperopia, astigmatism, and anisometropia were all significantly more frequent in children with than without amblyopia.

\section{Discussion}

Most previous studies defined amblyopia using strict VA criteria, failing to take into account the impact of treatment, $^{16-22}$ and thus making comparison of the true prevalence of amblyopia across studies difficult.

Nonetheless, our $1.9 \%$ prevalence, which takes into account all children previously diagnosed and treated with the condition regardless of their present VA, is still lower than reported rates from most previous studies. ${ }^{23-25}$ Using similar methodologies, we recently documented an almost identical prevalence $(1.8 \%)$ in a large $(n=1740)$ representative sample of younger, predominantly

Table 2 Treatment status and visual acuity outcomes (by cause) of 44 children diagnosed with amblyopia

\begin{tabular}{lcc}
\hline Cause & & Outcome \\
\cline { 2 - 3 } & $\begin{array}{c}\text { Proportion treated } \\
\mathrm{n}(\%)\end{array}$ & $\begin{array}{c}\text { Mean best-corrected } \\
\text { VA in logMAR letters } \\
\text { (CI) }\end{array}$ \\
\hline Anisometropia $(n=18)$ & $17(94.4)$ & $44.6(38.9-50.3)$ \\
Strabismus $(n=11)$ & $11(100.0)$ & $50.8(47.1-54.6)$ \\
Mixed $(n=10)$ & $6(66.7)$ & $40.3(32.6-47.9)$ \\
High ametropia $(n=4)$ & $3(75.0)$ & $42.4(38.2-46.6)$ \\
Unknown cause $(n=1)$ & $0(0.0)$ & $26.1(25.8-26.5)$ \\
All $(n=44)$ & $37(84.1)$ & $44.5(40.8-48.3)$ \\
\hline
\end{tabular}

aBest-corrected visual acuity $<6 / 12$.

LogMAR VA, logarithm of minimum angle of resolution visual acuity; CI, confidence interval. 
Table 3 Selected ocular outcomes in children with $(n=44)$ and without $(n=2309)$ amblyopia

\begin{tabular}{|c|c|c|c|}
\hline Ocular outcome & $\begin{array}{c}\text { Children with } \\
\text { amblyopia }(\mathrm{n}=44)\end{array}$ & $\begin{array}{l}\text { Children without } \\
\text { amblyopia }(\mathrm{n}=2309)\end{array}$ & P-value \\
\hline Mean SER, dioptres (SE) & $+1.47(0.66)$ & $+0.48(0.10)$ & 0.1 \\
\hline Mean cylinder, dioptres (SE) & $-1.32(0.19)$ & $-0.33(0.01)$ & $<0.0001$ \\
\hline \multicolumn{4}{|l|}{ Refractive error ( $n, \%)$} \\
\hline Myopia (SER $\leq-0.50 \mathrm{D})$ & $12(27.9)$ & $285(12.4)$ & 0.003 \\
\hline Hyperopia (SER $\geq+2.00 \mathrm{D})$ & $22(51.2)$ & $93(4.1)$ & $<0.0001$ \\
\hline Astigmatism (Cyl $\geq 1.00 \mathrm{D})$ & $19(44.2)$ & $199(8.7)$ & $<0.0001$ \\
\hline Anisometropia ( $\geq 1.00 \mathrm{D})$ & $29(67.4)$ & $67(2.9)$ & $<0.0001$ \\
\hline Spectacle use $(n, \%)$ & $29(65.9)$ & $419(18.2)$ & $<0.0001$ \\
\hline Strabismus $(n, \%)$ & $23(52.3)$ & $41(1.8)$ & $<0.0001$ \\
\hline Abnormal stereoacuity ( $<120$ arc seconds) & $28(68.3)$ & $59(2.6)$ & $<0.0001$ \\
\hline Noncorrectable visual impairment $^{\mathrm{a}}(n, \%)$ & $12(27.3)$ & $7(0.3)$ & $<0.0001$ \\
\hline
\end{tabular}

abest-corrected visual acuity $<6 / 12$.

$\mathrm{SE}$, standard error; SER, spherical equivalent refraction; D, dioptre; Cyl, cylinder.

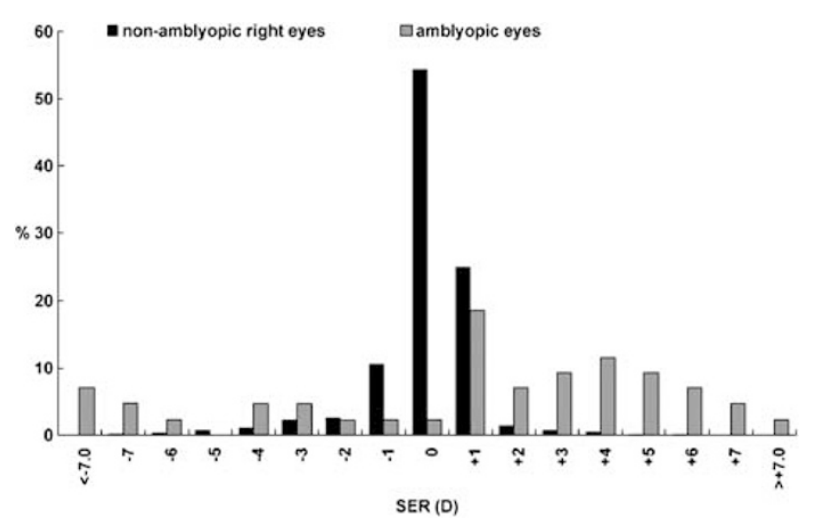

Figure 2 Distribution of spherical equivalent refraction (SER), expressed in dioptres (D), in 44 amblyopic eyes and 2309 nonamblyopic right eyes. Refraction of the right eye is used in four cases of bilateral amblyopia.

6-year-old Australian children. ${ }^{9}$ The proportion of previously detected amblyopia was somewhat higher in this older sample (89\%) than in the younger population $(72 \%)$, although most cases of amblyopia had already been diagnosed in both age groups. Correspondingly, the prevalence of non-correctable visual impairment (best-corrected $\mathrm{VA}<6 / 12$ ) owing to amblyopia was low in both samples, 0.5 and $0.9 \%$, for the older and younger age groups, respectively.

Snowdon and Stewart Brown's review of the literature on preschool vision screening ${ }^{5}$ prompted substantial debate about its place and efficacy, ${ }^{7,26-29}$ with controversy concerning the best screening tests, ${ }^{30}$ the timing and frequency of screening ${ }^{31}$ and likely cost-effectiveness. ${ }^{32,33}$ There are currently no routine vision screening programs in place in Australia, and the onus is largely on parents to have their child's vision tested either by a general practitioner or optometrist. Vision screening by orthoptists and nurses is provided on a smaller scale in some community health centres, although usage again relies on parental self-referral.

To our knowledge, this is the first study to evaluate the impact of amblyopia and its treatment on vision in a population-based sample and at an age beyond which treatment is unlikely to be beneficial. Our findings show that despite the lack of a routine vision-screening programme, the prevalence of non-correctable visual impairment owing to amblyopia is very low (0.5-0.9\%) in Australia, a consistent finding now across two large, representative samples of 6- and 12-year-old children. Two previously screened samples of Danish and Swedish school children each reported a $1.1 \%$ corresponding rate. $^{34,35}$

Comparison with a previously unscreened sample of elderly Australians from an area west of Sydney (Blue Mountains Eye Study) points to a possible improvement in the detection and treatment of amblyopia over the intervening $50-70$ years; $3.9 \%$ of people in that sample were diagnosed with amblyopia, $74 \%$ of whom were visually impaired in their affected eye. ${ }^{36}$ Thus, in the Blue Mountains sample, prevalence of visual impairment resulting from amblyopia was $2.9 \%$, more than 5 -fold greater than in the current study. Similarly, prevalence of unilateral amblyopia in another previously unscreened sample of older Australian adults was 3.1\%, with 54\% being visually impaired in the affected eye. ${ }^{20} \mathrm{It}$ is conceivable that improvements in antenatal care and infant health have led to a reduction in the incidence of amblyopia in recent decades. This might in part explain the lower prevalence of amblyopia in our childhood sample than that of older adults in the Blue Mountain Eye Study. Secondly, the diagnosis of amblyopia is likely 
to be more reliable in childhood. It is possible that subtle unrecognised eye disease is more easily misclassified as amblyopia in older adults.

We noted slight, but clinically significant, differences in the VA of children with different types of amblyopia. Children with strabismic amblyopia had the best outcomes, with mean best-corrected Snellen equivalent acuity of $6 / 7.5$. Corresponding mean corrected acuities for children with anisometropic and mixed amblyopia were $6 / 9$ and 6/12, respectively. Treatment outcome depends on a number of factors, including age at presentation, initial acuity, and compliance with treatment. ${ }^{37}$ Strabismic amblyopia is likely to be detected (and therefore treated) earlier than other types of amblyopia $^{36,38}$ because strabismus may be externally visible to the parents. On the other hand, some types of amblyopia may be inherently more refractory to treatment than others with some suggestion that visual outcomes from mixed amblyopia may be worse than from isolated anisometropic or strabismic amblyopia. ${ }^{37}$

Strengths of this study are its population-based design and uniform examination protocol. As mentioned, most previous amblyopia studies have not taken into account the impact of treatment. Although corroborating historical data were used to ascertain previously treated cases, this was done rigorously to minimise misclassification. For example, parental report of occlusion therapy needed to be consistent for affected and treated eyes, although the duration of occlusion therapy needed to be compatible with amblyopia treatment (i.e. weeks to months) rather than days, as for a traumatic eye injury.

The study also has some limitations. We had no information on the initial VAs of children with amblyopia, the intensity of the prescribed treatment regimen, or the children's compliance, all of which are important determinants of final VA outcomes.

Although the project achieved a reasonable response rate, $24.7 \%$ of the target population was not examined because parents did not give consent. Collection of information on socio-demographic factors, eye disease, and parental attitudes towards eye care in this group was limited by logistic barriers. Although overt participation bias was not noted, some of these children could still have significant visual loss from amblyopia that was undiagnosed or had failed treatment. Generalisation of our findings to the entire population therefore, needs to be made with caution.

Nevertheless, if previously treated children were less likely to participate in the study, we would have expected many more previously undetected cases of amblyopia in our sample. This was not the case; the majority of amblyopic children in our sample had been previously diagnosed with the condition, and this fact had not discouraged their parents from agreeing to their child's participation. There is also no reason to suspect that previously undiagnosed children would have selectively declined to participate, as lack of diagnosis by an eye practitioner by definition requires lack of parental awareness of the condition.

In addition, the high rates of detection and treatment seen in this sample may not necessarily apply to all Australian children, because access to and availability of eye care services as well as knowledge of common childhood eye conditions might vary geographically. Although most of the children in our sample had not participated in preschool vision screening programs, their parents appeared to have a low threshold for seeking professional eye care services by alternate means. This important characteristic may not be present in other unscreened populations. Notwithstanding the above limitations, this report provides a snapshot of the real world impact of amblyopia detection and treatment in a large Australian sample and in the context of nonmandatory vision screening.

\section{Acknowledgements}

The Sydney Myopia Study (Sydney Childhood Eye Study) is supported by the National Health and Medical Research Council (Grant No. 253732), the Westmead Millennium Institute, University of Sydney and the Vision Co-operative Research Centre.

\section{References}

1 Foran S, Wang JJ, Mitchell P. Causes of visual impairment in two older population cross-sections: the Blue Mountains Eye Study. Ophthalmic Epidemiol 2003; 10: 215-225.

2 Hrisos S, Clarke MP, Wright CM. The emotional impact of amblyopia treatment in preschool children: randomized controlled trial. Ophthalmology 2004; 111: 1550-1556.

3 Choong YF, Lukman H, Martin S, Laws DE. Childhood amblyopia treatment: psychosocial implications for patients and primary carers. Eye 2004; 18: 369-375.

4 Pediatric Eye Disease Investigator Group. A randomized trial of atropine $v s$. patching for treatment of moderate amblyopia in children. Arch Ophthalmol 2002; 120: 268-278.

5 Snowdon SK, Stewart-Brown SL. Preschool vision screening. Health Technol Assess 1997; 1: i-iv, 1-83.

6 Eibschitz-Tsimhoni M, Friedman T, Naor J, Eibschitz N, Friedman Z. Early screening for amblyogenic risk factors lowers the prevalence and severity of amblyopia. J AAPOS 2000; 4: 194-199.

7 Kvarnstrom G, Jakobsson P, Lennerstrand G. Visual screening of Swedish children: an ophthalmological evaluation. Acta Ophthalmol Scand 2001; 79: 240-244.

8 Robaei D, Rose K, Ojaimi E, Kifley A, Huynh S, Mitchell, P. Visual acuity and the causes of visual loss in a populationbased sample of 6-year old Australian children. Ophthalmology 2005; 112: 1275-1282. 
9 Robaei D, Rose KA, Ojaimi E, Kifley A, Martin FJ, Mitchell, P. Causes and associations of amblyopia in a population-based sample of 6-year old Australian children. Arch Ophthalmol 2006; 124: 878-884.

10 Scheiman MM, Hertle RW, Beck RW, Edwards AR, Birch E, Cotter SA et al. Randomized trial of treatment of amblyopia in children aged 7 to 17 years. Arch Ophthalmol 2005; 123: 437-447.

11 Wu C, Hunter DG. Amblyopia: diagnostic and therapeutic options. Am J Ophthalmol 2006; 141: 175-184.

12 Ojaimi E, Rose KA, Morgan IG, Smith W, Martin FJ, Kifley A et al. Distribution of ocular biometric parameters and refraction in a population-based study of Australian children. Invest Ophthalmol Vis Sci 2005; 46: 2478-2754.

13 Robaei D, Rose K, Kifley A, Mitchell P. Patterns of spectacle use in Australian school children: findings from a population-based study. J AAPOS 2005; 9: 579-583.

14 Huynh SC, Kifley A, Rose KA, Morgan I, Heller GZ, Mitchell P. Astigmatism and its components in 6-year old children. Invest Ophthalmol Vis Sci 2006; 47: 55-64.

15 Klein R, Klein BE, Linton KL, De Mets DL. The beaver dam eye study: visual acuity. Ophthalmology 1991; 98: 1310-1315.

16 Downing AH. Ocular defects in 60,000 selectees. Arch Ophthalmol 1945; 33: 137-143.

17 Cole RBW. The problems of unilateral amblyopia: a preliminary study of 10,000 national health patients. BMJ 1959; 1: 202-206.

18 Yassur Y, Yassur S, Zaifrani S, Sachs U, Ben-Sira I. Amblyopia among African pupils in Rwanda. B J Ophthalmol 1972; 56: 368-370.

19 Lithander J. Prevalence of amblyopia with anisometropia or strabismus among schoolchildren in the Sultanate of Oman. Acta Ophthalmol Scand 1998; 76: 658-662.

20 Brown SA, Weih LM, Fu CL, Dimitrov P, Taylor HR, McCarty CA. Prevalence of amblyopia and associated refractive errors in an adult population in Victoria, Australia. Ophthalmic Epidemiol 2000; 7: 249-258.

21 Ross E, Murray AL, Stead S. Prevalence of amblyopia in grade 1 schoolchildren in Saskatoon. Can J Pub Health 1977; 68: 491-493.

22 Helveston EM. The incidence of amblyopia ex anopsia in young adult males in Minnesota in 1962-63. Am J Ophthalmol 1965; 60: 75-77.

23 Ohlsson J, Villarreal G, Sjostrom A, Cavazos H, Abrahamsson M, Sjostrand J. Visual acuity, amblyopia, and ocular pathology in 12- to 13-year-old children in Northern Mexico. J AAPOS 2003; 7: 47-53.

24 Hopkisson B, Clarke JR, Oelman BJ. Residual amblyopia in recruits to the British army. BMJ (Clin Res Ed) 1982; 285: 940.
25 Preslan MW, Novak A. Baltimore vision screening project. Ophthalmology 1996; 103: 105-109.

26 Hartmann EE, Dobson V, Hainline L, Marsh-Tootle W, Quinn GE, Ruttum MS et al. Preschool vision screening: summary of a task force report. Ophthalmology 2001; 108: 479-486.

27 Kemper AR, Fant KE, Badgett JT. Preschool vision screening in primary care after a legislative mandate for diagnostic eye examinations. South M J 2003; 96: 859-862.

28 Bowman RJ, Williamson TH, Andrews RG, Aitchison TC, Dutton GN. An inner city preschool visual screening programme: long-term visual results. B J Ophthalmol 1998; 82: 543-548.

29 Stewart-Brown SL, Snowdon SK. Evidence-based dilemmas in pre-school vision screening. Arch Dis Child 1998; 78: 406-407.

30 Schmidt P, Maguire M, Dobson V, Quinn G, Ciner E, Cyert L et al. Comparison of preschool vision screening tests as administered by licensed eye care professionals in the Vision In Preschoolers Study. Ophthalmology 2004; 111: 637-650.

31 Williams C, Northstone K, Harrad RA, Sparrow JM, Harvey I. Amblyopia treatment outcomes after screening before or at age 3 years: follow up from randomised trial. BMJ 2002; 324: 1549 .

32 Joish VN, Malone DC, Miller JM. A cost-benefit analysis of vision screening methods for preschoolers and school-age children. J AAPOS 2003; 7: 283-290.

33 Gandjour A, Schlichtherle S, Neugebauer A, Russmann W, Lauterbach KW. A cost-effectiveness model of screening strategies for amblyopia and risk factors and its application in a german setting. Optom Vis Sci 2003; 80: 259-269.

34 Ohlsson J, Villarreal G, Sjostrom A, Abrahamsson M, Sjostrand J. Visual acuity, residual amblyopia and ocular pathology in a screened population of 12-13-year-old children in Sweden. Acta Ophthalmol Scand 2001; 79: 589-595.

35 Jensen H, Goldschmidt E. Visual acuity in Danish school children. Acta Ophthalmol (Copenh) 1986; 64: 187-191.

36 Attebo K, Mitchell P, Cumming R, Smith W, Jolly N, Sparkes R. Prevalence and causes of amblyopia in an adult population. Ophthalmology 1998; 105: 154-159.

37 Woodruff G, Hiscox F, Thompson JR, Smith LK. Factors affecting the outcome of children treated for amblyopia. Eye 1994; 8: 627-631.

38 Woodruff G, Hiscox F, Thompson JR, Smith LK. The presentation of children with amblyopia. Eye 1994; 8 623-626. 